\title{
PATTERN -BASED WITH SURFACE-BASED MORPHOMETRY SURVEY ON BRAIN CHANGES
}

\author{
R.Keerthana ${ }^{1}$, M.Babu ${ }^{2}$, S.R.Sridhar ${ }^{3}$ \\ ${ }^{I}$ PG Scholar (M.E), Department of Computer science, Knowledge Institute of Technology, Salem, Tamil Nadu, India \\ ${ }^{2}$ Assistant Professor, Department of Computer science, Knowledge Institute of Technology, Salem, Tamil Nadu, India \\ ${ }^{3}$ Assistant Professor, Department of Computer science, Salem, Tamil Nadu, India
}

\begin{abstract}
Morphometry is identifying and characterizing differences and correlations between brain shapes among population. Study of brain shape has drawn attention among many researchers on different diseases counting Schizophrenia, dyslexia, autism, Alzheimer and turner's syndrome. Many approaches have been proposed for computer-assisted diagnostic taxonomy. Several significant progressive brain changes occur during aging. Pattern-Based morphometry is a robust application for measuring the change in brain parametric mapping. By reason of bias featuring in image along with the algorithm, have a penalty term and inverse consistency that are necessary to oversight the change in non-biological structure. Morphometric analysis forces a pact between the sensitivity and specificity that has drawn reporting or increasing attention in the field of biological science. A novel technique illustrating the brain tissue aging survey with surface-based and multivariate pattern of morphometric brain change To keep the robustness and specificity contributed by the spatial term and cortical analyses, while maintaining the localization and sensitivity Experimental results propose a greater inter variability within normal aging as well as the generation of more sensitive based morphometry in brain survey.
\end{abstract}

Keywords: Image registration, Multivariate analysis, Cortical analysis, Pattern based morphometry (PBM), Surface based Morphometry (SBM).

\section{INTRODUCTION}

Medical Imaging with Biomedical Engineering has become an important component in our day to day clinical applications .A survey on detecting change in non -biological structure is a challenging task which can be either longitudinal or crossscale[4]. Estimations of survey are dependent by many factors .Some factors are with the images and others with its methods. There are different forms of analysis of brain such as Singlephoton emission computed tomography (SPECT), Computed tomography (CT), positron Emission Tomography (PET), and Magnetic resonance imaging (MRI) ,in which it is widely used for analysis. These are used to detect abnormality localization in patients. They are subjected to noise while segmentation. The major difficulty in brain change detection is to model change in terms of deformation at region boundaries from the boundary-based method. Morphometry of whole brain to detect abnormalities has approaches like Voxel-based to quantify mass-univariate or multivariate analyses and Tensor-Based Morphometry (TBM) [1] obtained from registration to measure the amount of deformation to measure the anatomical features of the subject. The TBM deals with the sensitivity and specificity with reference to the brain changes. Deformation varies in different ways (i.e.) the amount of deformation depends on the morphology of the subject.

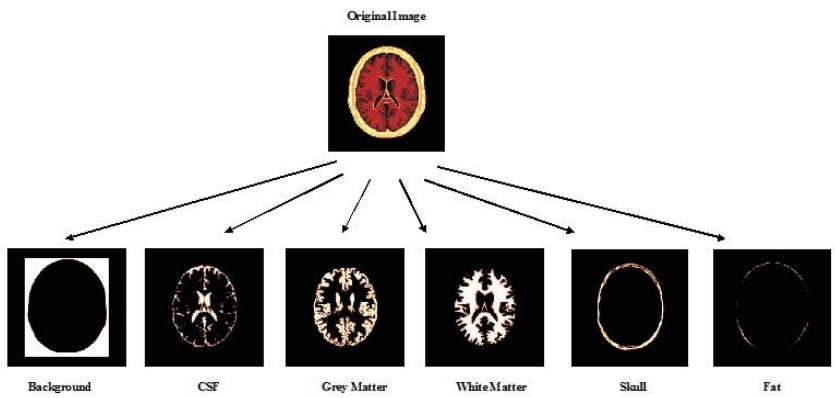

Fig-1 Six regions present in brain image usually used for morphometric analysis.

In this paper we will exploit a new technique Pattern-Based Morphometry (PBM) which a data driven technique. It uses sparse dictionary learning algorithm that is used to identify the differences globally. This is done along with another method Surface-based Morphometry (SBM) [4] which measures from geometric models of cortical surfaces.

\section{RELATED WORK}

The specificity of region-based approach along with the boundary-based approach were used to combine the localization and sensitivity advantages. By using TBM we can find the volume changes typically that appear at tissue 
boundaries in homogenous brain regions[1]. Brain registration is also one of the tools used for studying morphometry. Registration can be difficult depending on anatomical variability and complexity in structures. Log-jacobian images of such warps should be uniformly close to zero[1].

Multiple atlases of brain MRI is collected across subjects and computed. For atlases OASIS (Open Access series of Imaging Studies) database is used that range from 18 to $96[15]$.

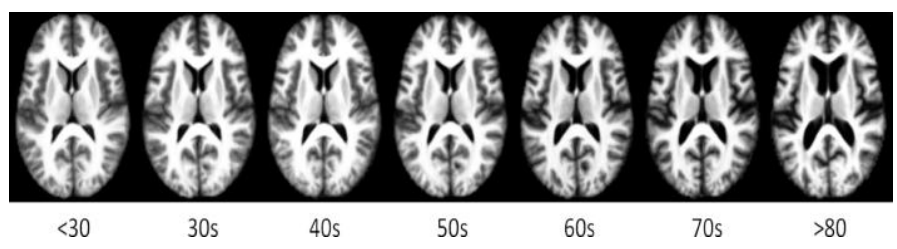

Fig-2 Atlases across different ages for subjects in the OASIS data set.

The atlases compute sharpness and features of image to higher degree. A method to overlay the images from their source and the manipulation of their transparency attributes or by assigning them to different color channels

Image fusion can be performed at three different levels 1.Pixel/data level, 2.Feature/attribute level and 3.Symbol/decision level. These are done to serve for different purposes. Fusion rule is being used here to determine the fusion results[12].

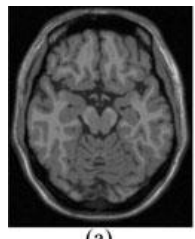

(a)

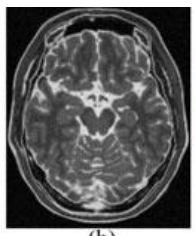

(b)

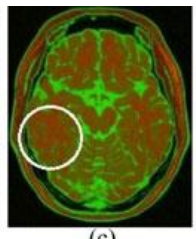

(c)

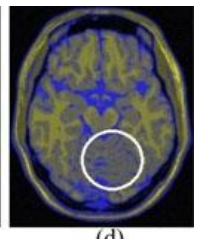

(d)
Fig-3 Overlaying monochrome images using different color channels.

Multistructure deffeomorphic registration approach to approve accuracy and robustness[2]. Many Group-based Neuroanatomical studies is the extraction of morphometric features that can be used for characterization of anatomical variability across or within groups .This study has approaches like voxel-based and tensor-based. Voxel-based morphometry computes the changes in volume due to registration[6]. It generates specific hypothesis about brain changes overtime. It is a automated technique that has grown in popularity .It uses statistics to identify difference in brain anatomy between collections of subjects, which in turn can be used to assume the presence of atrophy or, less normally, tissue development in subjects with disease.

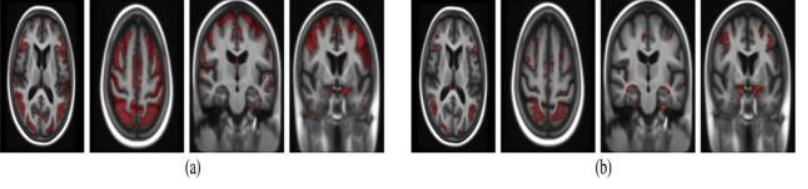

Fig-4.Visualization of the residual variance from group wise registration of all subjects in the OASIS database

Tensor-based morphometry is done in deformation fields. TBM uses the log-determinant jacobians. Studies the longitudinal changes. The image determines the multistucture and MRI-based approach.

Elevated specific absorption rate (SAR) associated with increased main magnetic field strength remains a major safety concern in ultra -high-field (UHF)[14]. SAR calculation requires the knowledge of electric field induced, and the local electrical properties of tissues.

Cortical thickness estimation performed[8] in-vivo via MRI is a technique to understand the progression of neurodegenerative diseases. Longitudinal results for control and $\mathrm{AD}$ (Alzheimer Disease ) subjects are done by three methods[4] .1.Free surfer, 2.laplacian, 3.Registration . Free Surfer cortical thickness pipeline processing involves intensity normalization, registration segmentation and automatic topology correction. Laplacian method segments Gray matter(GM), white Matter(WM) and cerbro spinal fluid (CSF) is done in T1 weighted image of tissue type. Registration method calculates WM, GM \& CSF segmentation and a greedy diffeomorphic registration was being used. Various methods are used to compute deformation of brain.

\section{METHOD}

Methods used to analyse are pattern-based and surface-based. Pattern-based morphometry (PBM) is a data driven approach. It uses a dictionary learning algorithm to extract global patterns that characterize group differences. Surface-based analysis derives morphometric measures from geometric models of the cortical surface.

Algorithm used for Pattern-based with surface-based morphometry (P-S BM) has the following steps 1.Changes in synthetic images 2.Surface Extraction 3.K-Singular value decomposition 4.Reconstruction 5.Group analysis.

\subsection{Step-1 Changes in Synthetic Images}

Large set of different images is generated by subtracting an image in Group 1(G1) from its neighbor Group 2 (G2). G1 contains set of images $\mathrm{M}=\{\mathrm{a} 1 \ldots \mathrm{am}\}$ and $\mathrm{G} 2$ contains set of images $\mathrm{N}=\{\mathrm{b} 1 \ldots \mathrm{bn}\}$, then the image is generated as

$$
\mathrm{GOI}=\mathrm{M}-\mathrm{N} \text { (for all am \& bn images) }
$$


Any image generated by subtracting an image in Group 1 from its neighbor in Group 2 can be expressed as a straight combination of a dictionary of image patterns that extricate the two groups. It is done to discover this dictionary of image patterns

\subsection{Step-2 Surface Extraction}

Layer of cortical surface is being measured and extracted. The cortical surface has the grey matter, white matter and CSF (cerebrospinal fluid) .Many manipulations are applied to the surface

\subsection{Step-3 K-Singular Value Decomposition}

It solves a matrix decomposition problem to extract a dictionary of $\mathrm{k}$ patterns from $\mathrm{X}$ which is taken from by Aharon,M.,Elad,M.,and Brukstein et.al. Along with this SVD approach thickness and weight of brain is being measured using the surface based analysis. The K-SVD approach here extracts the grey matter volume, white matter volume and CSF (cerebrospinal fluid) volume.

\subsection{Step-4 Reconstruction}

Image is being smoothed by the moving average smoothing technique and the image is being reconstructed.

\subsection{Step-5 Group Analysis}

Once the reconstruction has been computed, a group analysis is performed across $\mathrm{AD}$ control groups with the following 1.White matter 2.Grey matter 3.Cerebro Spinal Fluid 4.Thickness 5.Weight and ranked accordingly with pattern of images which is compared with age and gender of the control groups.

\section{EXPERIMENTAL RESULTS}

It is done to discover this dictionary of image patterns.

The identification is done using MAT lab.

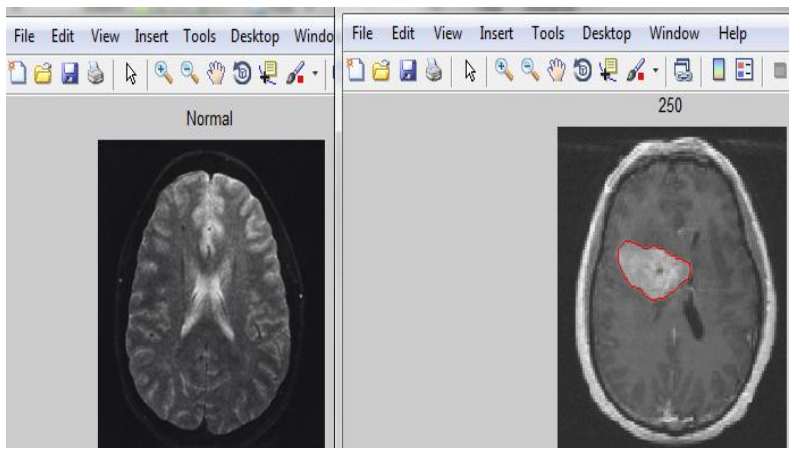

Fig-5. Image generated by subtracting an image using ADNI database images.
The time taken to identify single image is 8.765559 seconds.

The cortical surface has the grey matter, white matter and CSF (cerebrospinal fluid) as in fig 6.

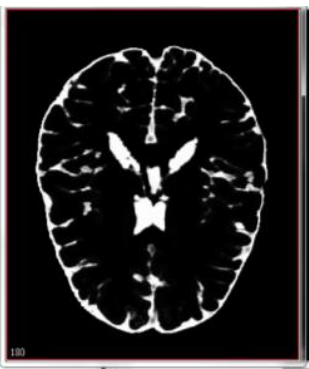

Fig-6 a)CSF

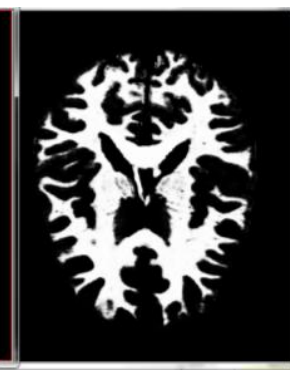

b)white matter

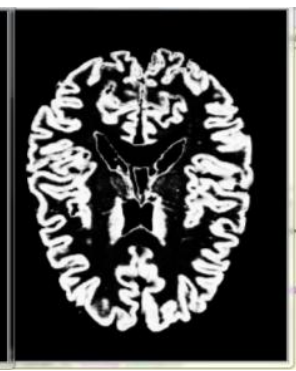

c)Grey matter
The K-SVD approach here extracts the grey matter volume, white matter volume and CSF (cerebrospinal fluid) volume. This is done when the surface extracted images are being analyzed. Image is being smoothed by the moving average smoothing technique and the image is being reconstructed. Pattern of images which is compared with age and gender of the control groups.

\section{CONCLUSIONS}

The technique of PBM with SBM measures both volume and surface of MR images and computes the inverse consistency of it and identifies across five control groups leaving the penalty term. Sensitivity has been increased along specificity and localization. Results are to be analyzed Future result can be on $3 \mathrm{D}$ brain images.

\section{ACKNOWLEDGEMENTS}

Data used in the preparation of result were obtained

[1]. Alzheimer's disease Neuroimaging Initiative (ADNI) database (www. loni. ucla. edu/ADNI). ADNI www.loni.Ucla.edu/ADNI/Collaboration/ADNICitatationshtm 1

[2]. http://www.bic.mni.mcgill.ca/brainweb.

\section{REFERENCES}

[1]. Evan Fletcher, Alexander Knaack, Baljeet Singh, Evan Lloyd, Evan Wu, Owen Carmichael, Charles DeCarli and Alzheimer 's Disease Neuroimaging Initiative. "Combining Boundary-Based Methods With Tensor-Based Morphometry in the Measurement of Longitudinal Brain Change" IEEE Transactions On Medical Imaging, Vol. 32, No. 2, February 2013223

[2]. Ali R. Khan, LeiWang\$, and Mirza Faisal Beg\$, "Multistructure Large Deformation Diffeomorphic Brain Registration," IEEE Transactions On Biomedical Engineering, Vol. 60, No. 2, February 2013 
[3]. Bilwaj Gaonkar, Kilian Pohl, and Christos Davatzikos, "Pattern Based Morphometry," Section for Biomedical Image Analysis, University of Pennsylvania.

[4]. Douglas N. Greve, Martinos, "An Absolute Beginner's Guide to Surface- and Voxel-based Morphometric Analysis," Center for Biomedical Imaging, Massachusetts General Hospital Harvard Medical School.

[5]. Emily L. Dennis, BA; Paul M. Thompson, PhD," Typical and atypical brain development: a review of neuroimaging studies"

[6]. Jennifer L. Whitwell, "Voxel-Based Morphometry: An Automated Technique for Assessing Structural Changes in the Brain," The Journal of Neuroscience, Department of Radiology, Mayo Clinic, Rochester, Minnesota 55905, August 5, 2009 • 29(31):9661-9664 • 9661.

[7]. J.-F. Mangin*, D. Rivière, A. Cachia, E. Duchesnay, Y. Cointepas, D. Papadopoulos-Orfanos, D. L. Collins,

A. C. Evans, and J. Régis, "Object-Based Morphometry of the Cerebral Cortex",IEEE Transactions On Medical Imaging, Vol. 23, No. 8, August 2004

[8]. Matthew J. Clarkson, M. Jorge Cardoso, Gerard R. Ridgway, Marc Modat, Kelvin K. Leung, Jonathan D. Rohrer, Nick C. Fox, Sébastien Ourselin, "A comparison of voxel and surface based cortical thickness estimation methods," Centre for Medical Image Computing (CMIC), The Engineering Front Building, University College London, London, WC1E 6BT, UK, Dementia Research Centre, UCL Institute of Neurology, University College London, London, WC1N 3BG, UK, NeuroImage 57 (2011) 856-865

[9]. Mitsuhiro Yoshita, MD, PhD, Evan Fletcher, PhD, and Charles DeCarli, MD, "Current Concepts of Analysis of Cerebral White Matter Hyper intensities on Magnetic Resonance Imaging"

[10]. M. Vatsa, R. Singh, and A. Noore, "Denoising and Segmentation of 3D Brain Images," IIIT Delhi, India, \{mayank, rsingh\}iiitd.ac.in, West Virginia University, Morgantown, USA, afzel.noore@mail.wvu.edu

[11]. Naftali Raz, Karen M. Rodrigue , Neuroscience and Biobehavioral Reviews 30 (2006) 730-748 www.elsevier.com /locate /neubiorev "Differential aging of the brain: Patterns, cognitive correlates and modifiers" Department of Psychology and Institute of Gerontology, Wayne State University, 87 East Ferry St., 226 Knapp Building, Detroit, MI 48202, USA

[12]. Rui Shen, Member, IEEE, Irene Cheng, Senior Member, IEEE, and Anup Basu, Senior Member, IEEE, "Cross-Scale Coefficient Selection for Volumetric Medical Image Fusion," IEEE Transactions On Biomedical Engineering, Vol. 60, No. 4, April 20131069

[13]. Simon S. Keller ${ }^{1} \&$ Neil Roberts ${ }^{2}$ "Measurement of brain volume using MRI: software, techniques, choices and prerequisites"

1) Clinical Research Imaging Centre (CRIC), Queen's Medical Research Institute (QMRI)University of Edinburgh email: kellers@liv.ac.uk
2) Division of Medical and Radiological Sciences, School of Clinical Sciences and Community Health, University of Edinburgh, UK

[14]. Xiaotong Zhang, Sebastian Schmitter, Pierre-François Van de Moortele, Jiaen Liu, and Bin He, Fellow IEEE, "From Complex B1 Mapping to Local SAR Estimation for Human Brain MR Imaging Using Multi-Channel Transceiver Coil at 7T" IEEE Transactions On Medical Imaging, Vol. 32, No. 6, June 2013

[15]. Yuchen Xie, Jeffrey Ho, and Baba C. Vemuri, Fellow, IEEE, "Multiple Atlas Construction From A Heterogeneous Brain MR Image Collection" IEEE Transactions On Medical Imaging, Vol. 32, No. 3, March 2013 